\title{
Procedurally related differences in the aggressive behavior of Betta splendens (Regan)
}

\author{
MICHAEL L. LOBB and GARVIN MCCAIN \\ University of Texas at Arlington, Arlington, Texas 76019
}

\begin{abstract}
The agonistic behaviors of male Betta splendens (Regan) were studied during both mutually viewing and mutually caged encounters in an investigation of differences between these types of encounters. With the pairings of animals held constant, the experience of prior mutual viewing which produced significant habituation did not significantly alter aggression in subsequent mutually caged encounters. Furthermore, animals which exhibited submissiveness (zero display rates) during mutually caged encounters significantly increased the intensity of aggression during subsequent mutual viewing, while the dominant caged mates decreased the rate of displays. These results were interpreted to mean that mutual viewing produces habituation while mutual caging produces conditioned suppression, and that generalizations from one situation to the other may therefore be unwarranted.
\end{abstract}

On the basis of Simpson's (1968) findings, Peeke and Peeke (1970) have criticized the use of mirror images as releasing stimuli in the study of Betta splendens (Siamese fighting fish). They suggest that the sequential and spatial relationships which characterize Betta aggressive behavior outside of the laboratory would be for the most part precluded by the use of mirror images. This argument may be extended to include not only the obvious case of models as agonistic stimuli, but also to the mutual viewing (MV) technique in which animals are separated by a transparent screen. At first, the MV technique would appear to be a more realistic analog of the natural situation, since it permits the establishment of some sequential relationships among agonistic behaviors, but separation by a glass screen would prevent the occurrence of primary aversive stimulation (pain) which results from strikes and tail beatings among mutually caged $B$. splendens. Furthermore, the absence of such attacks would preclude the establishment of sequential relationships between attack and other agonistic behaviors. For these reasons, the validity of generalizations from MV to mutual caging (MC) conditions would appear to be questionable.

In addition to the questionable validity of generalization from MV to MC conditions, there is some question about the use of mutual viewing as a technique. Typically, the MV procedure involves the use of two animals, a test fish and a stimulus fish, separated by a transparent screen. The display rate of the test fish is generally ignored. The finding of sequential relationships (Simpson, 1968) between the display of mutually responding $B$. splendens and the indication of positive correlations between the rates of these displays (Gallagher, Herz, \& Peeke, 1972 , p. 364) strongly suggests that the display rates of the test and stimulus fishes are interdependent.
Thus, any interpretation which may have been based upon observations of a test fish would have been drawn without consideration of how these observations might have been modulated by the stimulus fish. The point is that aggression in an unrestricted encounter apparently involves the physical interaction of two antagonists as opposed to an isolated test fish viewing a stimulus fish through a glass screen. This apparent difficulty with technique could be overcome by making simultaneous recordings of the display rates between paired animals.

The purpose of the following three experiments was, first, to assess the extent to which mutual viewing can attenuate display rates as compared to mutually caged conditions and, second, to examine possible differences in the intensity and pattern of displays between these conditions. To compensate for the apparent procedural problems of previous studies, our data were drawn from concurrent observations of both members of paired Siamese fighting fish.

\section{EXPERIMENT I}

The purpose of manipulations in this experiment was to assess the level of intensity of aggressive behavior after 14 days of constant MV and to determine the extent of response diminution by comparing the observation periods.

\footnotetext{
Method

Subjects. Eight male B. splendens were purchased in Sattihip, Thailand, where all experimentation was conducted. These animals were 50 to $65 \mathrm{~mm}$ in length and had fully developed fins and the coloration common to adult members of this species. All of the subjects used in this report were of the hybrid strain of $B$. splendens which are distinctively different from the wild brown fish common to this region.

Apparatus. The aquariums consisted of cylindrical glass jars,
} 
$90 \mathrm{~mm}$ in diameter $\times 115 \mathrm{~mm}$ in height. The perimeters of these jars were opaqued with the exception of a viewing window, which was $50 \mathrm{~mm}$ in height $\times 25 \mathrm{~mm}$ in width. Each home aquarium, which served also as a test apparatus, contained between 125 and $150 \mathrm{ml}$ of semidistilled water. This water was obtained by collecting drippings from window air conditioning units and was changed every 3 days. The ad-lib diet consisted of mosquito larvae. Constant lighting was provided by a 40 -W flourescent bulb. The temperature varied between $65^{\circ}$ and $70^{\circ} \mathrm{F}$, and although this was a low ambient temperature for these fish, it was maintained as a constant condition access experimental conditions.

Procedure. In the first experiment, displays were measured after 14 days of continuous mutual viewing. The first 10-min MV test involved the same pairing of animals as has had been maintained during the 14-day period, while the second 10-min MV test was conducted with rearranged pairs.

The animals were randomly divided into three groups (one for each experiment) and into pairs within each group. In these experiments, the groups were tested using pairs of animals so that each stimulus animal served also as a test animal and the data were concurrently collected on both members of each pair. The data was recorded by means of a tape recorder. In this procedure, the experimenter recited each behavior as it was observed and indicated the identification number of the animal that had exhibited that behavior. For purposes of brevity, the behaviors were coded by their initials. As soon as an animal's number and the initials of the behavior it had executed had been recited, the observer would attend to the conspecific. In this manner, the observer was continuously alternating his attention between the members of each pair throughout the duration of an encounter. The encounters consisted of $10 \mathrm{~min}$ of observation per pair of animals, and the pairs within each group were observed in sequence. After all of the experimentation had been conducted, the tapes were replayed and the data transcribed.

Based, in part, on descriptions by Forselius (1957) and by Simpson (1968) and, in part, on observations conducted during pilot studies, four agonistic and three avoidance behaviors were chosen for examination during the MV encounters. The avoidance behaviors consisted of perpendicular orientation, lateral withdrawal, and rapid swimming, while the agonistic behaviors included frontal orientations, lateral displays, gill cover erection, and tail beating.

An avoidance behavior was considered to have occured if an animal exhibited a perpendicular orientation (PO) with respect to the surface of the water. Such perpendicular orientations are commonly called appeasement (Forselius, 1957). Since appeasement may decrease the incidence of aversive stimulation, it is here assumed to be a behavior by which aversive stimulation is avoided. Active avoidance behaviors of a spatial nature such as rapid swimming (RS) and lateral withdrawal (LW) were also recorded. Rapid swimming was identified on the basis of the exceptionally high rate of locomotion in response to an antagonist's approach. Lateral withdrawal was identified by a characteristically slow withdrawal in a perpendicular direction away from the approaching antagonist. Lateral withdrawal was also characterized by a considerably reduced medial fin erection.

In the MC condition, the measurements of lateral displays. (LD), gill cover erections (GCE), and avoidance behaviors were identical to those employed during the MV periods. Since, in the MC condition, animals can and frequently do vary their spatial orientation, a judgment of frontal orientation (FO) must inevitably differ procedurally between $\mathrm{MV}$ and $\mathrm{MC}$ encounters. In the mutually caged situation, a FO was identified if an animal turned to face the conspecific, approached with medial fins erected and mouth open, and appeared to track the antagonist's movements. If these movements were followed by a rapid charge at and contact with the antagonist, a strike was considered to have occurred and a count of one was recorded. On some occasions, several strikes occurred in rapid succession without fully executing all of the motions of FO in which case a count of one was made for each strike by the attacking animal.

The procedures employed with all three experiments are shown in Table 1. The subjects of the first experiment consisted of four pairs. Mutual viewing was initiated by turning the glass jars of a pair so that the viewing uindow's were adjacent and viewing was interrupted only to change the water, which was accomplished without netting or handling the animals. On the 14th day, MV was discontinued for $1 \mathrm{~h}$ prior to collecting data from the first data period.

Subsequent to data collection for the first period, MV was again interrupted for $1 \mathrm{~h}$ while the pairs were rearranged. During the second 10-min encounter, the animals of each pair were viewing a novel conspecific.

\section{Results and Discussion}

Following 14 days of continuous MV, the fish continued to display. The mean number of GCE's per $10 \mathrm{~min}$ of observation was 1.5 , while FOs and LDs occurred at mean rates of 4.75 and 0.75 , respectively. The conclusion supported by these data is

Table 1

Procedures

\begin{tabular}{c}
$\begin{array}{c}\text { Continuous MV } \\
\text { Original Pairs }\end{array}$ \\
\hline 14 days \\
MC; Encounters \\
Original Pairs
\end{tabular}

Six 10-min encounters with an 8-h interencounter interval

$\frac{\begin{array}{c}\text { Visual } \\ \text { Isolation }\end{array}}{1 \mathrm{~h}}$

Isolation

$24 \mathrm{~h}$ at 6-h intervals

\section{Experiment I}

\begin{tabular}{ccc}
$\begin{array}{c}\text { Experiment I } \\
\text { MV; Original } \\
\text { Pairs }\end{array}$ & & $\begin{array}{c}\text { Visual } \\
\text { Isolation }\end{array}$ \\
\hline $\begin{array}{c}10 \mathrm{~min} / \text { pair } \\
\text { Experiment II } \\
\text { MV; Original } \\
\text { Pairs }\end{array}$ & & $1 \mathrm{~h}$ \\
\hline $\begin{array}{l}\text { Four 10-min viewing periods } \\
\text { at 6-h intervals }\end{array}$ & & \\
Isolation \\
\hline
\end{tabular}

\begin{tabular}{c}
$\begin{array}{c}\text { MV; Rearranged } \\
\text { Pairs }\end{array}$ \\
\hline 10 min/pair \\
$\frac{\text { MV; Rearranged }}{\text { Pairs }}$ \\
\hline $\begin{array}{l}\text { Four 10-min viewing } \\
\text { periods at 6-h intervals }\end{array}$
\end{tabular}
periods at 6 -h intervals

\section{Experiment III}


simply that 14 days at continuous mutual viewing is not sufficient to completely wane aggression. By implication, the MV procedure, regardless of length of presentation, may not produce zero aggressive responding. Furthermore, the introduction of novel stimulation through the rearrangement of pairs resulted in an increase in the display rate. The difference in display rates between conditions was statisically tested by application of a t-test for mean deviation (Md) between matched pairs of data points (Hays, 1963; p. 335). The increases both in FO $(\mathrm{Md}=+13.25, \mathrm{t}=3.07, \mathrm{df}=4)$ and $\mathrm{LD}$ (Md $=+4.5, \mathrm{t}=2.88, \mathrm{df}=4$ ) were found to be significant $(\mathrm{p}<.05)$, while the increase in GCE was not significant $(\mathrm{Md}=+.414)$. Thus, the introduction of novel stimulation is sufficient to reinstate attenuated display rates. That these display rates increased indicates that the attenuation process was at least in part specific to the particular antagonist which had been provided.

\section{EXPERIMENT II}

The purpose of the second experiment was to assess the effects of $\mathrm{MC}$ encounters on subsequent MV encounters and to compare the extent of response diminution between procedures. In these manipulations, the pairings were held constant, however, in a subsequent test of stimulus specificity, the pairings were altered. Finally, the data of Experiment II were analyzed by separately examining dominant and submissive subgroups instead of the usual procedure of analyzing only the data of test animals.

\footnotetext{
Method

Subjects. The subjects were 10 naive $B$. splendens with essentially identical characteristics to those described in Experiment $I$.

Apparatus. The apparatus was the same as in Experiment II.

Procedure. The five pairs of subjects of the secorad experiment were first subjected to $\mathrm{MC}$ encounters, then to $\mathrm{MV}$ encounters with the same pair arrangements, and finally to $\mathrm{MV}$ encounters with pairs rearranged. The MC manipulation consisted of six 10-min encounters with an interencounter interval of $8 \mathrm{~h}$. During this procedure, an encounter would begin by pouring the contents of one aquarium, including the subject, into a conspecific's aquarium and adjusting the water level to about $150 \mathrm{ml}$. This procedure was adopted because handling by netting had suppressed results in earlier pilot work. After $10 \mathrm{~min}$ of observation, the pair would be separated by pouring one animal into an empty jar. The initiation and termination of encounters was thus accomplished without handling or netting the animals. During all interencounter intervals, the pairs were physically and visually isolated. A 24-h interval intervened between the last MC encounter and the first MV encounter. The first series of four MV encounters was conducted with the same pair arrangements as had been used during the MC encounters. These encounters were begun by rotating the viewing windows of a pair until they were adjacent for $10 \mathrm{~min}$, after which the viewing was interrupted. These encounters were equally spaced over a 24 -h period, following which the pairs were visually isolated for $24 \mathrm{~h}$. The pairs were then rearranged so that during the next four MV encounters each animal viewed a novel conspecific from the same group. These last four MV encounters were conducted to obtain an
}

indication of the extent to which the previous encounters had sufficed to diminish the display rate.

\section{Results and Discussion}

The data collected from observation during the second experiment were analyzed by examining the differential display rates between members of each pair under each of the three experimental conditions. The purpose of this analysis was to assess the contribution of each conspecific to the data. This was accomplished by first inspecting the results of the sixth and final MC encounter so as to identify the dominant member of each pair. An animal was judged to be the dominant member if it had exhibited a higher rate of strikes, FOs, LDs, GCEs, TBs, and a lesser rate of avoidance behavior than its conspecific. The data collected on these dominant animals was then pooled and is shown under the title of Dominant Conspecifics in Figure 1a, $1 \mathrm{~b}$, and $1 \mathrm{c}$. The data from the remaining five animals (one from each pair) were presented under the title of Submissive Conspecifics. In Figure 1a, the combined frequency of attack (strike + FOs + TBs) has been depicted for both the dominant and the submissive subgroups. Similarly, threat (GCE + LD) and avoidance frequencies have been depicted in Figures $1 \mathrm{~b}$, and 1c, respectively. The t-test for Md's between matched pairs of data points was used to assess the existence of statistical significance between subgroups within each experimental condition, e.g., MV, MC, novel mutual viewing. A one-tail t-test (Hays, 1963, p. 320) was also used for the difference in mean deviations between experimental conditions.

As can be seen from Figure 1a, both subgroups were attacking at an equal rate during the first $\mathrm{MC}$ encounter; however, these rates diverged significantly $(\mathrm{Md}=106, \mathrm{SD}=5.95, \mathrm{t}=4.24, \mathrm{df}=4, \mathrm{p}<.05)$ over encounters until the submissive subgroups was exhibiting at a near zero level. The change in experimental conditions from MC to MV of the same pairs resulted in a decrease in the attack rate for the dominant subgroup and an increase in the attack of the submissive subgroups. The difference in Mds between these conditions was statistically significant $(\mathrm{Md}=66.22, \mathrm{SD}=31.38, \mathrm{t}=2.11$, $\mathrm{df}=4, \mathrm{p}<.05$ ). The subsequent $\mathrm{MV}$, or rearranged pairs, resulted in a significant increase in the attack rate of the dominant subgroup, while that of the submissive subgroup increased slightly but not significantly $(\mathrm{Md}=67.5, \mathrm{SD}=8.77, \mathrm{df}=8, \mathrm{t}=7.70$, $\mathrm{p}<.05$ ) and in rearranged pairs were significantly different $(\mathrm{Md}=107.25, \mathrm{SD}=14.13, \mathrm{t}=8.17$, df $=4, p<.01$ ).

The results of observation of threat which are depicted in Figure $1 \mathrm{~b}$ are highly similar to the attack data. During early MC encounters, both subgroups significantly diverged $(\mathrm{Md}=25.17, \mathrm{SD}=23.46$, $t=2.40$, df $=4, p<.05)$. By the fourth encounter, the submissive subgroup was attacking and threaten- 

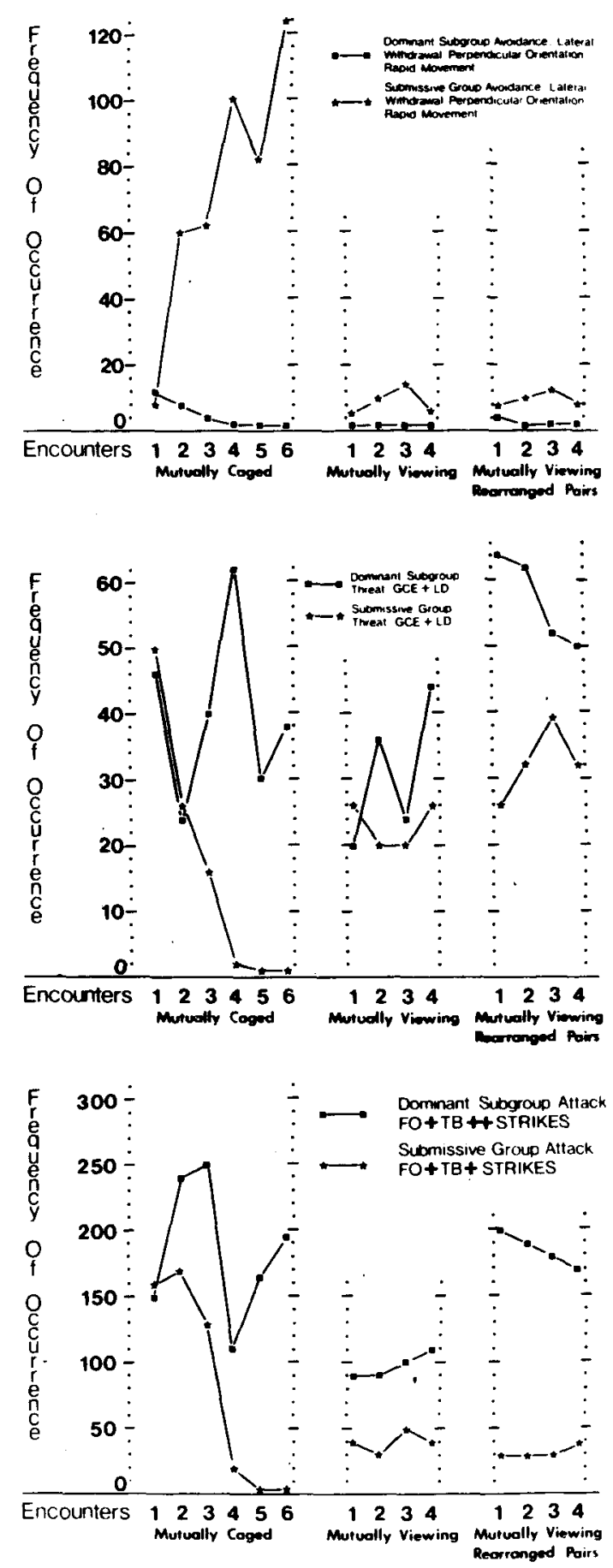

Figure 1. (a) Attack behavior, Experiment II. (b) Threat behavior, Experiment II. (c) Avoidance behavior, Experiment II.

ing at a near zero level, in contrast to the subjects of Experiment I, which continued to display after 14 days of constant mutual viewing. A change in experimental conditions from MC to MV of the same pairs resulted in a significant increase $(\mathrm{Md}=18.250$, $\mathrm{t}=5.22$, df $=8, \mathrm{p}<.01$ ) in the threat rate of submissive animals, while a rearrangement of pairs under MV conditions resulted in a significant increase
$(\mathrm{Md}=+19.250, \mathrm{t}=2.49, \mathrm{df}=8, \mathrm{p}<.025)$ in the threat rate of the dominant subgroup.

The findings of the second experiment warrant several conclusions. First, with respect to submissive conspecifics, the MC procedure is a sufficient condition for producing zero attack and threat rates, while the MV procedure is not; the reverse sequence, i.e., MV then MC, was performed in Experiment III, and those results further support this conclusion. The attenuation of attack and threat rates during MV encounter is due to a progressive decrease in rate among the submissive animals while the dominant ones continue to attack and threaten at about the same rate over encounters. Second, MV following $\mathrm{MC}$ encounters is not sufficient to maintain the zero rates achieved during the MC condition (the MC-MV sequence of Experiment II). Additionally, the agonistic rates of dominant conspecifics instantly declined, which indicates that such rates may be technique specific. Third, the introduction of novel conspecific is sufficient for significantly increasing attack and threat rates of dominant but not of submissive animals.

The results of observations on avoidance behavior in the second experiment appears in Figure 1c. The first notable difference between avoidance and agonistic behavior is that during the initial MC encounters the rate of avoidance is quite low in comparison to the relatively high initial rates of attack and threat. Over the course of MC encounters, the rates of avoidance diverge significantly $(\mathbf{M d}=70.50$, $\mathrm{SD}=42.50, \mathrm{t}=3.71, \mathrm{df}=4, \mathrm{p}<.05$ ) among the subgroups; this is due to a progressive increase in avoidance on the part of the submissive subgroup. The subsequent shift in experimental procedure from MC to MV of the same pairs resulted in a significant and immediate decrease in the avoidance rate of the submissive animals $(\mathrm{Md}=67.75, \mathrm{SD}=23.784$, df $=4, t=2.76, p<.025$ ), and this rate did not change following the rearrangement of pairs. Throughout these procedures, the dominant subgroup continued to exhibit a zero or near-zero level of avoidance. These findings warrant the conclusion that avoidance is always exclusively a behavior of the submissive subgroup, which progressively develops during the MC encounters. Apparently, avoidance is also a technique-specific behavior in that it occurs under MC conditions but not under MV conditions.

\section{EXPERIMENT III}

The primary purpose of the third experiment was to assess the effect of prior MV on subsequent $\mathrm{MC}$ encounters by examining for differences in the frequency and pattern of agonistic displays between these conditions. Additionally, this experiment was designed to assess the possibility of reinstatement between phases of mutual viewing as a control for the possibility of a reinstatement between $\mathrm{MV}$ and 
MC phases. Thus, three MV phases were followed by a MC phase.

\begin{abstract}
Method
Subjects. The subjects were 10 naive $B$. spendens with essentially identical characteristics to those described in Experiment $I$.

Apparatus. The same apparatus was again used.

Procedure. The first MV phase consisted of four encounters dispersed over a 20 -h period followed by $23 \mathrm{~h}$ of visual isolation. The test for reinstatement was conducted in the second MV phase, which consisted of six MV encounters dispersed over a 24-h period. Thus, two reinstatement tests were made prior to initiating the MC phase. A 28-h interval of visual isolation intervened between the last MV encounter and the MC phase. Four MC encounters were conducted during a $10-\mathrm{h}$ period.

Unlike Experiment II, the data collected in the third experiment were analyzed without regard to within-pairs differences, since this technique was previously demonstrated in Experiment II. That is, the data from Experiment III was summed across each pair and within each encounter. This procedure represents an analysis of the data without isolating the contribution of the stimulus animal from the test animal.
\end{abstract}

\section{Results and Discussion}

The results of MV encounters of the third experiment, which were collected in three phases, appear in Figure 2. The record of mutually viewing bouts among the same pairs, which are depicted in Figure 2 , indicate a considerable decrease in the frequency of agonistic behaviors. This decrease was tested with a one-tail $t$-test for Mds between matched pairs of data points (first and second) and was found to be significant at the .05 level (FO: $M d=-30.3$, $\mathrm{t}=6.34, \mathrm{df}=8$; $\mathrm{LD}: \mathrm{Md}=-3.2, \mathrm{t}=2.16, \mathrm{df}=8$ ). In the second phase, the rate of FO decreased slightly while the combined rate of GCE and LD (threat) underwent a small but insignificant increase. The rate of TB decreased to zero by the eighth session and was not observed again during the remainder of the MV sessions. During the third phase (Observation Periods 11 through 22), the rates of FO, LD, and GCE were essentially unchanged from the second phase. Two aspects of these preexperimental manipulations were important. First, these subjects had been given sufficient $\mathrm{MV}$ to allow for habituation to the specific stimuli of their conspecific prior to the introduction of the MC treatment. Second, the interval without agonistic stimulation between the last MV session and the first MC encounter was not a sufficient condition for reinstating the previously attenuated rate, since during each successive $\mathrm{MV}$ phase the rates did not increase.

During the subsequent four mutually caged encounters, the rate of $\mathrm{FO}(\mathrm{Md}=+68.9, \mathrm{t}=2.80$, $\mathrm{df}=4)$ and $\mathrm{LD}(\mathrm{Md}=+22.0, \mathrm{t}=2.49$, $\mathrm{df}=4)$ increased significantly $(p<.05)$. The measurement of GCE increased, but not significantly, which may have been due to the greater difficulty in measuring the accelerated response rates characteristic of $\mathrm{MC}$ encounters. These four MC encounters also differed markedly from the mutual viewing sessions, in that TB reappeared at a very high rate and that strikes

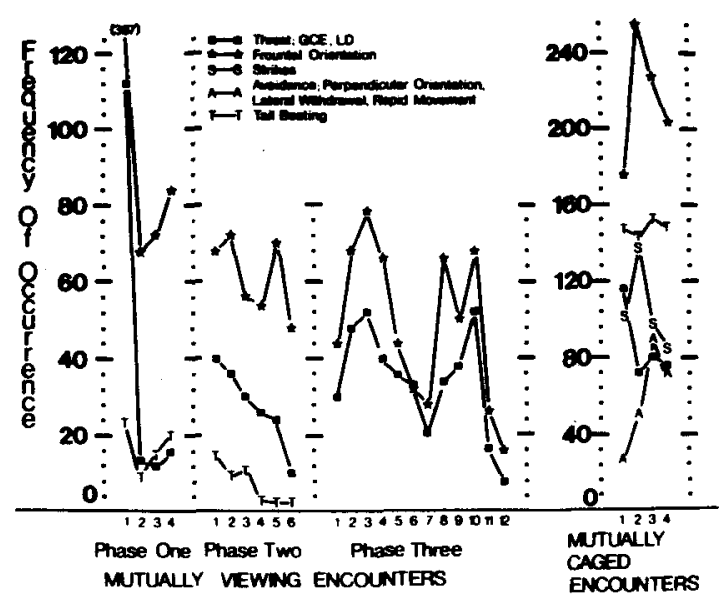

Figure 2. Agonistic behaviors, Experiment III.

appeared at an even higher rate. Furthermore, the avoidance behaviors which were absent from observations during MV encounters appeared in MC encounters.

These findings support three conclusions. First, the stimulus-specific habituation established through a MV procedure does not significantly affect the rates of agonistic behaviors during subsequent $\mathrm{MC}$ encounters, in which the same specific stimuli (conspecifics) are provided. Second, the differences in behaviors between MC and MV appear, at least in part, to be due to the presence of primary aversive stimulation resulting from TB and strikes. And third, avoidance appears to be specific to the mutually caged technique.

To further assess the effect of prior mutual viewing on subsequent mutually caged encounters of the third experiment, the mutually caged data of the third experiment was compared to the first four mutually caged encounters of the second experiment. Thus, mutually caged aggression with prior mutual viewing was compared to mutually caged aggression without prior mutual viewing. The difference in means for each behavioral category were not statistically significant. These comparisons further strengthen the conclusion that habituation which results from prior mutual viewing does not affect mutually caged aggression. Conclusions of habituation among aggressive conspecifics resulting from mutual viewing may thus be inapplicable to mutually caged conditions.

The possibility of differences in the pattern of agonistic behaviors between conditions was assessed by application of the Spearman rank order correlation technique. In Experiment III, the correlation for each behavior between $\mathrm{MV}$ and $\mathrm{MC}$ condition (FO, +0.31; LD, +0.24; GCE, +0.078) were of a low order and nonsignificant. In contrast, the correlation coefficients between behaviors but within MV and MC conditions were generally significant, based on the rank order of display rates. Within the 
MC condition of Experiment III, the ranks of animals according to frequency of GCE and FO were significantly. correlated (rho $=+0.55, \mathrm{t}=5.38$, $\mathrm{a}=.05$ ) as were the FO-LD (rho $=+0.75, \mathrm{t}=3.16$, $a=.05)$. In the Experiment II MV condition, the GCE-FO (rho $=+0.84, \mathrm{t}=4.42, \mathrm{a}=.05$ ) and the FO-OD (rho $=+0.77, \mathrm{t}=3.05, \mathrm{a}=.05$ ) were correlated. The correlation of GCE and LD within the MV condition was not significant (rho $=0.51$, $t=1.70$ ). These findings support the conclusion that the pattern of agonistic behaviors differs significantly between mutually caged and mutual viewing encounters. The same conclusion can be argued from the difference in aggression response frequencies between the submissive and dominant subgroups of Experiment II.

\section{GENERAL DISCUSSION}

Clayton and Hinde (1967) reported that after extended (10 days) intermittent mirror image stimulation, the GCE rate of $B$. splendens achieved an apparently asymptotic, nonzero level. Such a finding raises a question as to what procedure would be sufficient for a zero response rate. Peeke and Peeke (1970) have reported that massed trials produce lower rates than spaced trials, which suggests that extended massed training might suffice. However, in the first experiment, 14 days of constant MV did not result in zero rates, which suggests that visual stimulation and accompanying habituation lack the potency necessary for complete waning of aggression. In striking contrast, four 10-min MC encounters were sufficient for complete waning among submissive conspecifics. A habituation interpretation of the rate decrease among submissive animals would be critically limited by the finding that cagemates did not either habituate or substantially decrease the rate of agonistic behaviors. Presumably, habituation as a learning process would be common to both members of a pair of $B$. splendens.

The main effect of the present effort indicates that the composition and pattern of agonistic behaviors differ significantly between $M C$ and $M V$ encounters regardless of the sequence in which these encounters are arranged. These findings, along with the suddenness of behavioral change between conditions, suggest that the quality, quantity, and pattern of agonistic behaviors observed in these experiments were technique or procedure specific. The principle point of this paper is that interpretations such as habituation, which have been based on the results of MV procedures, may not warrant generalization to aggressive behavior in either the MC encounter or other situations.

The major procedural difference between MC and MV encounters appears to involve the lack of aversive stimulation during mutual viewing. The absence of pain-producing strikes and TB during MV limits such encounters to a habituation format, since the repeated stimulation of a response (FO, LD, GCE, etc.) is not followed by negative reinforcement, e.g., strikes and TB. While the MV encounter may be analogous to a habituation procedure, the MC encounter would appear to be best described as a conditioned suppression procedure. First, the necessary negative reinforcing stimuli are present, and second and most important, these reinforcers have been shown to be sequentially related to other responses. For example, a GCE by one animal is most frequently followed during early encounters by TB from the conspecific (Simpson, 1968). We may assume the hypothesis that strikes systematically follow FO and LD of the conspecific so that a conditioned suppression format is established. Furthermore, the conditioned inhibition or suppression hypothesis can account for the differential response rates between paired conspecifics of the present study, which as a finding cannot be handled by the habituation hypothesis.

Although the current literature on aggression attenuation has tended to favor the habituation hypothesis, some consideration should be given to Lorenz's (1966) avoidance hypothesis. In the present studies, the initial low rate of avoidance behaviors would not support a characterization of avoidance in the Siamese fighting fish as being wholly innate. Over encounters, the progressive decrease in agonistic behaviors and corresponding progressive increase of avoidance among submissive conspecifics may be best described as innate predispositions which are modified in rate by the experience factor. Thus, while, as Lorenz suggests, avoidance may be the major mechanism of aggression attenuation, the rate of avoidance necessary for successful attenuation may require conditioning in a $B$. splendens.

In a recent paper (Rhoad, Kalat, \& Klopfer, 1975), the dimunition of aggressive behavior in response to models, mirror images, and live stimulus animals has been interpreted in terms of associative learning. The growth of an active avoidance response is offered as the mechanism for this diminution and the habituation hypothesis appears insufficient. The Rhoad et al. paper, combined with the present report, would appear to strongly warrant a reappraisal of the habituation hypothesis.

\section{REFERENCES}

Clayton, F. L., \& Hinde, R. A. The habituation and recovery of aggressive displays in Betta splendens. Behaviour, 1967, 30, 96-106.

Forselius, S. Studies of anabantid fishes: I, II and III. Zoologica, Uppsala, 1957, 32, 93-597.

Gallagher, J. E., Herz, M. J., \& Peeke, H. V. S. Habituation of aggression: The effects of visual social stimuli on 
behavior between adjacently territorial convict cichlids (Cichlasoma nigrofasciatum). Behavioral Biology, 1972, 7, 359-368.

HaYs, W. L. Statistics for psychologists. New York: Holt, Rinehart and Winston, 1963.

Lorenz, K. Z. On aggression. New York: Harcourt, Brace and World, 1966.

Pavlov, I. P. Conditioned inhibition. In I. P. Pavlov, Conditioned reflexes. New York: Dover, 1960. Pp. 68-87.

Pefke, H. V. S., \& Peeke, S. C. Habituation of conspecific aggressive responses in the Siamese fighting fish (Betta splendens). Behaviour. 1970, 26, 232-245.

Rhoad, K. D., Kalat, J. W., Ke Klopfer, P. H. Aggression and avoidance by Betra splendens toward natural and artiticial stimuli. Animal Learning and Behavior, 1975, 3, 271-276.

Simpson, M. J. A. The display of the Siamese fighting fish, Betta splendens. Animal Behaviour Monograph, 1968, I (Whole Number).

(Received for publication August 8, 1975; revision accepted March 22, 1976.) 